\title{
Towards Guided Wave Robotic NDT Inspection: EMAT Size Matters
}

\author{
Oksana Trushkevych \\ Department of Physics \\ University of Warwick \\ Coventry, UK \\ O.Trushkevych@warwick.ac.uk
}

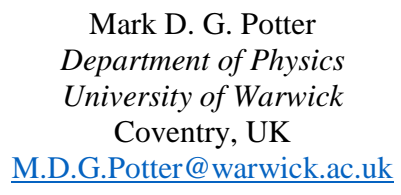

\author{
Steven Dixon \\ Department of Physics \\ University of Warwick \\ Coventry, UK \\ S.M.Dixon@warwick.ac.uk
}

\author{
Charles MacLeod \\ Department of Electronic \& \\ Electrical Engineering \\ University of Strathclyde \\ Glasgow, UK \\ charles.macleod@strath.ac.uk
}

\author{
Morteza Tabatabaeipour \\ Department of Electronic \& \\ Electrical Engineering \\ University of Strathclyde \\ Glasgow, UK \\ Morteza.tabatabaeipour@strath.a \\ c.uk
Anthony Gachagan
Electrical Engineering
University of Strathclyde
Glasgow, UK
a.gachagan@strath.ac.uk \\ Department of Electronic \&
}

\author{
Gordon Dobie \\ Department of Electronic \& \\ Electrical Engineering \\ University of Strathclyde \\ Glasgow, UK \\ gordon.dobie@strath.ac.uk
}

\author{
S. Gareth Pierce \\ Department of Electronic \& \\ Electrical Engineering \\ University of Strathclyde \\ Glasgow, UK \\ s.g.pierce@strath.ac.uk
}

\author{
Rachel S. Edwards \\ Department of Physics \\ University of Warwick \\ Coventry, UK \\ R.S.Edwards@warwick.ac.uk
}

\begin{abstract}
The first steps towards fast robotic screening of wall thinning in the industrially relevant example of $10 \mathrm{~mm}$ thick steel plates are reported. Electromagnetic acoustic transducers (EMATs) are used to generate and detect guided shear horizontal wavemodes, as these show promise for this type of inspection. EMATs are miniaturised to reduce magnetic drag on ferritic steels, and are designed to produce $\mathrm{SH}_{0}$ and $\mathrm{SH}_{1}$ wavemodes with $22 \mathrm{~mm}$ wavelength, which is suitable for testing wall thinning in these samples. Miniaturisation and the resulting reduction of magnetic drag force allows the EMATs to be mounted on a small crawler robot which can then be used to scan the sample / structure.
\end{abstract}

\section{Keywords-EMAT, SH guided waves, NDT, robotic inspection}

\section{INTRODUCTION}

Ultrasonic guided waves are promising for fast screening of large areas. In particular, shear horizontal wavemodes are suitable for inspection of wall thinning in plates and pipes, with generation of this wavemode possible using electromagnetic acoustic transducers (EMATs) [1,2]. EMATs are well suited for generating guided waves in conducting samples, and their noncontact nature is promising in robotic inspection applications as they remove the need for couplant. Guided waves such as Lamb and $\mathrm{SH}$ modes have been compared for thickness gauging applications. The $\mathrm{SH}_{1}$ wavemode was identified as suitable for measuring wide-area gradual thinning in reflection and transmission [3]. Small flaws in welds can also be measured

This work was funded by EPSRC grant "UK Research Centre in NonDestructive Evaluation (RCNDE)” (EP/L022125/1). using the $\mathrm{SH}_{1}$ mode, with the mode excited in the region of low dispersion (see Figure 1) [4-6]. In the region of strong dispersion, $\mathrm{SH}_{1}$ and higher order modes will be sensitive to wall thinning, demonstrated through a change in arrival time, while the non-dispersive $\mathrm{SH}_{0}$ mode will show a consistent arrival time for a fixed EMAT separation when used in pitch-catch mode [7]. Wall thinning can additionally be identified through identifying the cut-off frequency for higher order SH modes [8]. A combination of inspection using cut-off frequency, changes in arrival time, mode conversions, and transmitted or reflected wave amplitudes is the most promising way forward for fast wall loss screening [2]. To implement this and to access the highly dispersive regime of the $\mathrm{SH}_{1}$ mode, a large wavelength must be chosen.

This work considers the design of new EMATs for automated inspection when used on a robotic crawler. Firstly, a suitable wavelength for inspection must be chosen, and the EMAT designed to generate at this wavelength. Secondly, standard SH wave EMAT designs contain a large number of magnets, and the magnetic drag can be large. The simple way to reduce magnetic drag is to put the EMATs into a holder on wheels and use rolling friction to slide them along a sample. For successful implementation with a small crawler robot, however, reducing drag this way is not sufficient, and hence the EMATs used here are miniaturised. Miniaturisation has a second benefit of offering higher spatial resolution when scanning, and higher sensitivity to defects with small lateral dimensions [9]. 


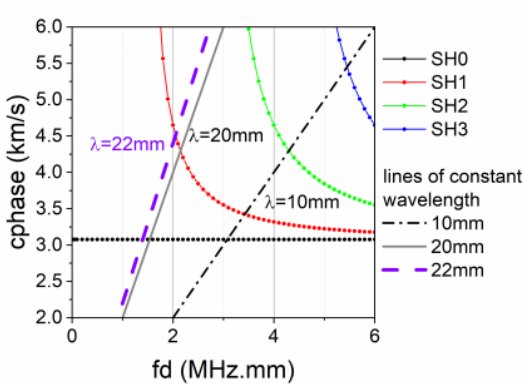

(a)

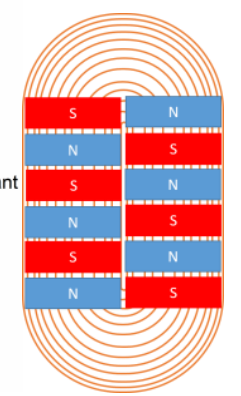

(b)
Fig. 1. (a) Dispersion curves for $\mathrm{SH}$ waves, produced for $10 \mathrm{~mm}$ thick carbon steel plate, together with lines of constant wavelength. (b) Standard PPMEMAT design: racetrack coil and magnets.

\section{DESIGN CONSIDERATIONS}

\section{A. Mode and wavelength selection - why large wavelength is necessary}

The choice of wavelength and frequency of operation is done through analysing phase speed dispersion curves for different modes. Fig 1(a) shows the first four SH modes together with lines of equal wavelength. Typical EMATs for SH waves use a periodic permanent magnet (PPM) array which sets a wavelength (Fig 1(b)); signals will be generated where the line for that wavelength crosses the dispersion curves, once the corresponding frequency is chosen [1]. Commercial EMATs are often built to produce a $10 \mathrm{~mm}$ wavelength. This does not allow generation of $\mathrm{SH}_{1}$ in $10 \mathrm{~mm}$ thick plates in the highly dispersive regime. Instead, here we use $22 \mathrm{~mm}$ wavelength. From the dispersion curves, the excitation should be around $200 \mathrm{kHz}$ to obtain the $\mathrm{SH}_{1}$ mode. Due to the broadband nature of the EMAT design used, and variability in samples (such as thin rust layers), the EMATs described here are excited at frequencies $160 \mathrm{kHz}$ and $200 \mathrm{kHz}$. At these excitation frequencies, the $\mathrm{SH}_{0}$ mode is also generated. The $\mathrm{SH}_{0}$ mode is non-dispersive and is not affected by wall thinning [2,7], hence it is a convenient reference, especially where there are any variations in amplitude or arrival time related to liftoff or transducer positioning that otherwise could be misinterpreted as defects.

\section{B. Transducer miniaturisation - testing the limits}

To build an EMAT with $22 \mathrm{~mm}$ wavelength using a standard design, the overall magnetic field strength would be large enough to create difficulties when inspecting ferritic steels, as the robotic crawler may not be able to move the EMATs. We explore the possibilities for reducing the number of magnets used in the traditional SH PPM-EMAT design. The smallest possible EMATs for use with small robots, once optimised for defect detectability on $10 \mathrm{~mm}$ steel, are made and tested experimentally.

Generation: the standard approach to using a PPM configuration is taken to the extreme minimum, with only 4 magnets used in total. The resulting generation EMAT footprint can then be as small as a wavelength: only $22 \mathrm{~mm}$ length $\mathrm{x} 22$ $\mathrm{mm}$ width, producing $22 \mathrm{~mm}$ wavelength (these are referred to as mini generators). A regular PPM-EMAT producing the same wavelength would be three times larger in both length and width, with around nine times more magnetic drag. A larger generation EMAT could be used where higher beam directivity is required (referred to as directional mini generators), as this may be necessary for long distance propagation and for avoiding beam spreading when mapping out structural features [10].

Detection: traditionally, matched PPM-EMATs are used for detection of SH waves. Here we use smaller detectors, with the same wavefront width and half the length of the miniaturised generation EMAT (referred to as mini detectors). The ultimate reduction in detector size was achieved by using small linear coils for sensing SH waves (referred to as micro detectors). This allows reduction of the size of the sensing transducer by 10 times, and as a result its magnetic drag can be reduced by up to 100 times. Both types of detectors (mini and micro) can be used in inspection, depending on the requirements of the system. Mini detectors offer significantly higher SNR, however, micro detectors offer excellent spatial resolution. All directivity measurements reported in this work (section IV) have been done using micro detectors, as this gave a higher level of detail compared to their larger counterparts.

\section{EMAT CHARACTERISATION}

\section{A. Modes produced and interaction with defects}

Fig 2 shows sonograms of the signal produced using the miniaturised EMATs (mini generator and micro detector) on steel, over a region of magnetite (thin rust layer) as well as on the same region of steel plate after the magnetite layer had been removed. The differences in generated modes are due to the complexities associated with magnetostriction generation mechanism. The $\mathrm{SH}_{0}$ and $\mathrm{SH}_{1}$ modes are generated with excellent signal to noise ratio (SNR) on magnetite at $160 \mathrm{kHz}$ using the mini generator and micro detector (Fig 2(a)). Without the magnetite layer, a weak, high dispersion $\mathrm{SH}_{1}$ is generated at

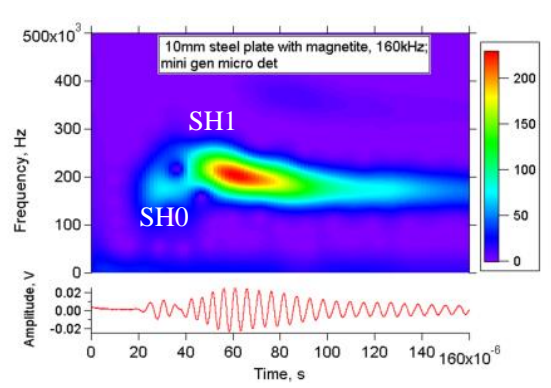

(a)

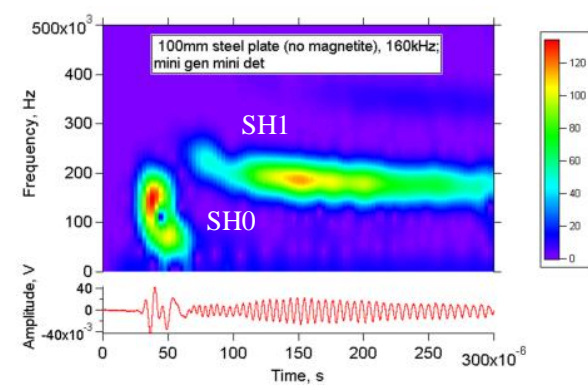

(b)

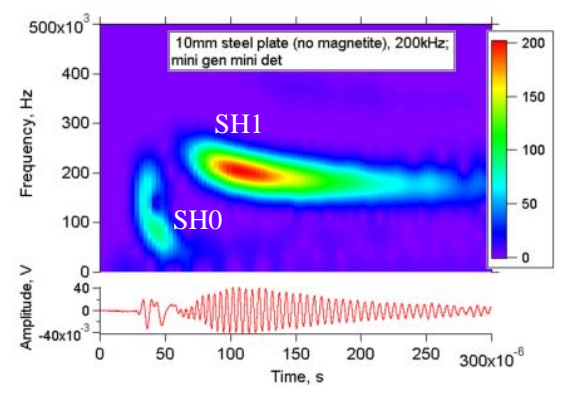

(c)

Fig 2. Frequency-time representation (sonogram) of the generated signal on $10 \mathrm{~mm}$ steel plate (a) with magnetite at $160 \mathrm{kHz}$ excitation by mini generator and micro detector; (b) no magnetite at $200 \mathrm{kHz}$ excitation, mini generator mini detector; 16 averages in all cases. 
(a)

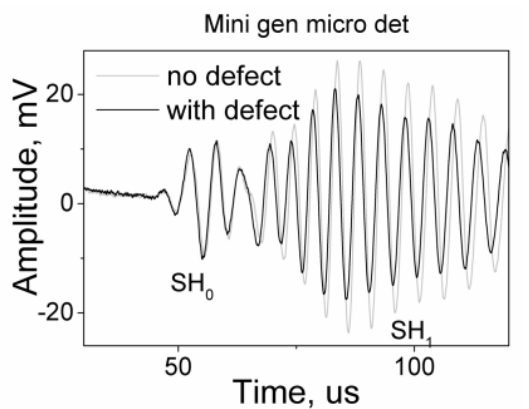

(b)

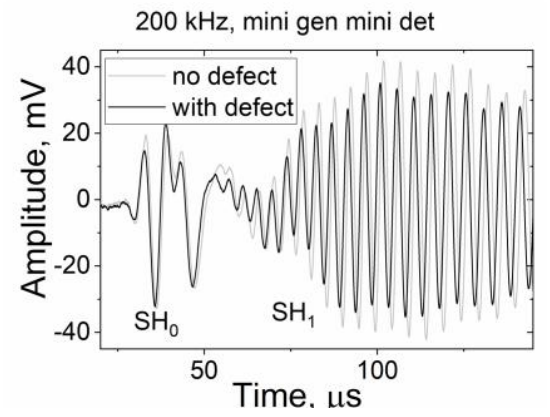

Fig. 3. Defect influence on SH1 mode (a) with magnetite layer at $160 \mathrm{kHz}$ excitation by mini generator and micro detector; (b) no magnetite at 200 $\mathrm{kHz}$ excitation, mini generator \& mini detector; 16 averages, defect is $20 \mathrm{~mm} \times 20 \mathrm{~mm}$ patch with $20 \%$ wall thinkness removed.

$160 \mathrm{kHz}$ on clear steel (Fig 2 (b)), and an optimal $\mathrm{SH}_{1}$ signal for wall thickness monitoring is produced at around $200 \mathrm{kHz}$ (Fig 2 (c)), as expected from the dispersion curves. To improve SNR without magnetite, the mini detector was used instead of the micro detector. The type of detector (mini vs micro) did not affect the modes generated and detected, only SNR.

Fig 3(b) shows signals on clear plate on an area with and without a small wall thinning patch, with the size $20 \times 20 \mathrm{~mm}$, where only $20 \%$ of wall thickness is removed. The $\mathrm{SH}_{1}$ wavemode produced in both cases is visibly affected by $20 \%$ reduction in wall thickness, with the patch close to the size of the wavelength used $(20 \mathrm{~mm} \times 20 \mathrm{~mm})$, with data shown in Fig 3.

In practice, the differences between clear and magnetite coated steel can be distinguished by the efficiency of EMAT generation. This knowledge will feed into strategies for analysing wall thinning, to take into account changes in the types of modes generated on rust/magnetite and the SNR. Testing frequencies could be changed dynamically during inspection to be optimally sensitive to wall thinning on any surface.

\section{B. Comparison with standard PPM-EMATs-SNR}

The expected tradeoff in making EMATs smaller is the reduction in SNR. Indeed, the miniaturised EMATs have a reduced SNR compared to commercial options, but SNR is sufficient for experiments, as shown by Fig 3. Mini EMATs can be operated in a more broadband manner, which gives flexibility in inspection. Figure 4 shows the comparison of signals obtained from a standard design (commercial) EMAT pair with $20 \mathrm{~mm}$ wavelength (Sonemat Ltd., model number SHG/D2031s) and from the miniaturised EMAT pairs with $22 \mathrm{~mm}$ wavelength. All measurements were conducted using 16 averages, with $30 \mathrm{~cm}$ between transducer centres, in the same position on a $10 \mathrm{~mm}$ thick steel plate with no magnetite on the surface.

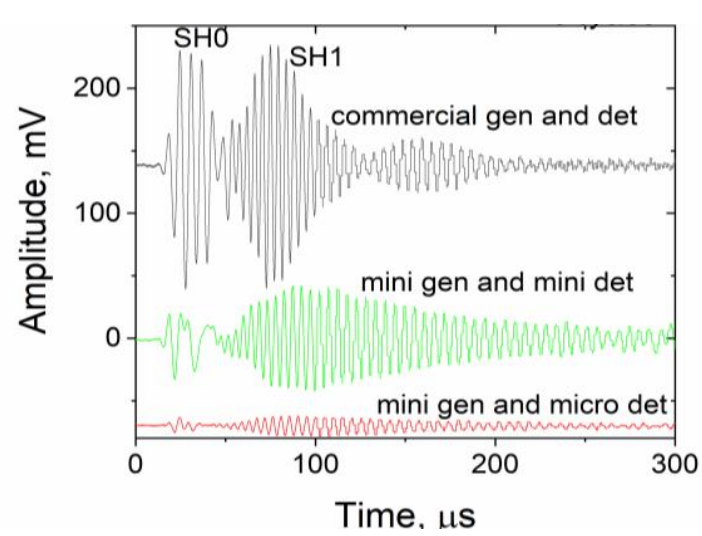

Fig. 4. (a) Signals produced at $200 \mathrm{kHz}$ using commercial EMATs with 20 mm wavelength, and miniaturised EMAT pairs: mini generator and mini dtetctor (middle trace) and mini generator and micro detector (bottom trace).

The differences in the shape of the observed signals are because the wavelengths of the transducers are slightly different $(22 \mathrm{~mm}$ for mini and $20 \mathrm{~mm}$ for commercial EMATs). At the same frequency of excitation, the modes generated will show a slightly different dispersion (see Fig 1). Commercial EMATs are narrowband due to the PPM design used, while the miniature EMATs are inherently broadband. This further contributes to the differences in signal shape. Despite these minor differences, the measurements allow for a meaningful comparison of SNR. The SNR values of the tested transducers for both $\mathrm{SH}_{0}$ and $\mathrm{SH}_{1}$ modes at $200 \mathrm{kHz}$ excitation are summarised in Table 1.

\section{Directivity}

Directivity of the miniaturized generating transducers has been measured. Directivity of a standard design EMAT has also been simulated at $227 \mathrm{kHz}$ generating $\mathrm{SH}_{1}$ mode [10]. Note that in simulations the detector is a single point. In the experiment we used the micro detector. Fig 5 (a) shows the experimental directivity of the mini generator, and Fig 5 (b) of the directional mini generator. Directivity is sufficiently good for wall loss screening in both cases. The directional mini generator has much lower beam spreading/better directivity and has a significant advantage at larger transducer spacing. It is also

Table 1 Signal to noise ratio comparison between a commercial pair of transducers and several combinations of miniaturised EMAT pairs at 200 $\mathrm{kHz}$. Magnetic attraction forces are specified for each pair, together with the drag due to magnetic attraction when placing the EMATs on wheels with $10 \mathrm{~mm}$ diameter. The max. pulling weight of the robot is $13.5 \mathrm{~kg}$.

\begin{tabular}{|c|c|c|c|c|c|}
\hline $\begin{array}{c}\text { EMAT } \\
\text { pair }\end{array}$ & $\begin{array}{c}\text { Ampl } \\
\text { ratio }\end{array}$ & $\begin{array}{c}\text { SNR } \\
\text { power }\end{array}$ & $\begin{array}{c}\text { SNR } \\
\text { dB }\end{array}$ & $\begin{array}{c}\text { Magnetic } \\
\text { forces (kg) }\end{array}$ & $\begin{array}{c}\text { Drag force } \\
\text { (kg) }\end{array}$ \\
\hline \multicolumn{4}{|c|}{$\mathrm{SH}_{1}, 200 \mathrm{kHz}, 30 \mathrm{~cm}$ distance } & $\begin{array}{c}\text { Est. } \\
\text { attraction, no } \\
\text { liftoff }\end{array}$ & $\begin{array}{c}\text { Est. drag } \\
\text { with rollers }\end{array}$ \\
\hline $\begin{array}{l}\text { Commerc. } \\
20 \mathrm{~mm}\end{array}$ & 62 & 3786 & 36 & 211 & 21.1 \\
\hline $\begin{array}{l}\text { Directional } \\
\text { mini gen } \\
\text { mini det }\end{array}$ & 26 & 673 & 28 & 38 & 3.8 \\
\hline $\begin{array}{l}\text { Mini gen } \\
\text { mini det }\end{array}$ & 26 & 686 & 28 & 22.8 & 2.3 \\
\hline $\begin{array}{l}\text { Mini gen } \\
\text { micro det }\end{array}$ & 5 & 25 & 14 & 16.4 & 1.6 \\
\hline
\end{tabular}


better suited to mapping [10]. The side lobes observed in the experiment can be resolved when using the micro detector, and can be attributed to wave interference due to magnetic edge effects. This is in line with the modelling [10].

\section{MAGNETIC DRAG AND ROBOTIC INSPECTION}

For implementation of EMATs onto a robot, the drag experienced by the robot is a significant consideration for potential applications. The estimation of drag force requires knowledge of the magnetic forces, the liftoff, the design of wheels used, and the coefficient of friction for the whole system. Note that it is standard practice of magnet manufacturers to quote the result of magnetic attraction calculations as the weight which could be held by the magnets, and it is common to specify robot pull force in $\mathrm{kg}$.

The magnetic forces were estimated using the magnet manufacturer's specifications [11], assuming the minimal possible liftoff of EMATs above the sample (zero liftoff was used in calculations here to account for the worst case scenario; the expected liftoff during operation is $0.1-0.5 \mathrm{~mm})$. The coefficient of friction depends on the diameter of wheels used; a roller with $10 \mathrm{~mm}$ diameter wheels is used here. We focus on the drag component due to magnetic attraction, as this has the largest effect. The mass of the transducers is not included for simplicity as this is a small addition, and the miniaturised transducers weigh less than standard design ones. A simplified calculation of drag using rolling friction can be done using $F_{d r a g}=k_{f r}\left(M_{g e n}+M_{d e t}\right)$, where $M$ is magnetic attraction force and

(a)

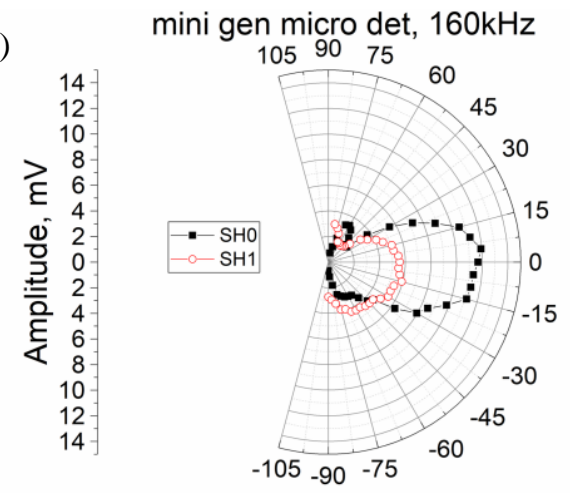

(b)

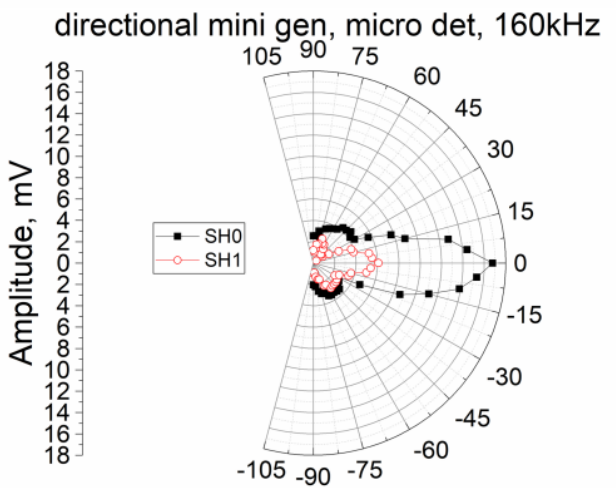

Fig. 5. Directivity of generating EMATs: (a) mini generator (160 kHz), (b) directional mini generator $(160 \mathrm{kHz})$. $k_{f r}$ is friction coefficient, which depends on wheel radius $r$ via $k_{f i}=c l / r\left(c_{l}=0.5 \mathrm{~mm}\right.$ for steel on steel); for $r=5 \mathrm{~mm}, k_{f r}=0.1$. The results of the calculations are given in Table 1 .

An Inuktun miniature magnetic crawler (MicroMag) is used in our work $[10,12]$. The pulling rate of the robot is specified by the manufacturer to be: normal pull $5.5 \mathrm{~kg}$; short term pull $9 \mathrm{~kg}$; peak pull:13.5 kg. The traditional EMAT pair therefore cannot be pulled by this robot as the drag force, even when using rolling friction, is $21.1 \mathrm{~kg}$ - above its operational level. All combinations of miniaturised EMATs can be pulled by the robot, as they are well within its operational range, enabling automated scanning of samples.

\section{CONCLUSIONS AND FURTHER WORK}

SH wave EMATs have been miniaturised for implementation onto a robotic inspection system for the industrially relevant case of measuring wall thinning in $10 \mathrm{~mm}$ thick ferritic steel. The SNR of the miniaturized EMATs is moderately reduced compared to the standard design commercial PPM-EMATs, but is within acceptable limits for operation. EMAT miniaturisation makes a significant difference in drag forces and enables the use of long wavelength EMATs on crawler robots.

\section{ACKNOWLEDGMENT}

This work was funded by the EPSRC "UK Research Centre in Non-Destructive Evaluation (RCNDE)" (EP/L022125/1), Core Research 2018-20, "Remote and Automated Delivery of NonContact NDE".

\section{REFERENCES}

[1] M. Hirao and H. Ogi, "An SH-wave EMAT technique for gas pipeline inspection", NDT \& E International, vol 32, 3, pp.127-132, 1999

[2] A.C. Kubrusly, M. A. Freitas, J. P. von der Weid and S. Dixon, "Interaction of $\mathrm{SH}$ guided waves with wall thinning", NDT\&E International, vol 101, pp. 94-103, 2019

[3] P Khalili and P Cawley, NDT and E International," The choice of ultrasonic inspection method for the detection of corrosion at inaccessible locations", vol 99, pp 80-92, 2018

[4] S Dixon, PA Petcher, Y Fan, D Maisey and P Nickolds, "Ultrasonic metal sheet thickness measurement without prior wave speed calibration.", J Phys D: Appl. Phys., vol 46 p445502, 2013

[5] PA Petcher, SE Burrows and S Dixon, "Shear horizontal (SH) ultrasound wave propagation around smooth corners" Ultrasonics, vol 54, pp 9971004,2014

[6] P. A. Petcher and S. Dixon, "Weld defect detection using PPM EMAT generated shear horizontal ultrasound," NDT \& E International, vol 74, pp. 58-65, 2015.

[7] JL Rose, Ultrasonic waves in solid media, Cambridge University Press 2014

[8] PA Petcher and S Dixon, "Mode mixing in shear horizontal ultrasonic guided waves", Nondestr Testing \& Eval vol 32 (2) pp113-132, 2017

[9] O Trushkevych and RS Edwards, "Characterisation of small defects using miniaturised EMAT system”, NDT\&E International 107102140 (2019)

[10] M Tabatabaeipour, O Trushkevych, G Dobie, RS Edwards, S Dixon, C MacLeod, A Gachagan and SG Pierce, "A feasibility study on guided wave-based robotic mapping", IEEE 2019 IUS Proceedings, under review

[11] https://www.supermagnete.de/eng/, accessed 2019

[12] http://inuktun.com/en/products/onsite-standard-products/micromagminiature-magnetic-crawler/, accessed 2019 